\title{
Effect of ulinastatin on cytokine reaction during gastrectomy
}

\author{
Ji Hun Park, Sang Hyun Kwak, Cheol Won Jeong, Hong Beom Bae, and Seok Jai Kim \\ Department of Anesthesiology and Pain Medicine, Chonnam National University Medical School, Gwangju, Korea
}

Background: Inflammation plays an important role in the postoperative morbidity of organs, which is related to the activation of pro-inflammatory and anti-inflammatory cytokines. Ulinastatin (Urinary trypsin inhibitor, UTI) is a serine protease inhibitor found in human urine or serum that inhibits the activation of human leukocyte elastase. This study examined the effect of UTI on the inflammation response in patients undergoing a gastrectomy.

Methods: Thirty patients scheduled to undergo a gastrectomy were divided into two groups as follows: Control group (untreated, $\mathrm{n}=15)$ and UTI group (100,000 units of UTI were continuously injected intravenously for 2 hours, $\mathrm{n}$ = 15). Arterial blood was sampled before surgery (T0), 10 minutes after its onset (T1), at its end (T2), and 1 hour after surgery (T3) to measure the level of cytokines.

Results: Both the control and treatment groups had higher interleukin (IL)-6 levels at T2 and T3 than T0, and the level increased with time. However, the increase was smaller in the treatment group. The IL-8 levels were not activated significantly in any of the groups.

Conclusions: UTI inhibits the secretion of IL-6, which is an inflammatory cytokine produced after a gastrectomy. This shows that UTI can decrease the inflammation reaction caused by surgical stress. (Korean J Anesthesiol 2010; 58: 334-337)

Key Words: Gastrectomy, Inflammatory cytokine, Interleukin-6, Urinary trypsin inhibitor.

Received: October 21, 2009. Revised: November 17, 2009. Accepted: February 18, 2010.

Corresponding author: Sang Hyun Kwak, M.D., Ph.D., Department of Anesthesiology and Pain Medicine, Chonnam National University Medical School, 671, Jebong-no, Dong-gu, Gwangju 501-757, Korea. Tel: 82-62-220-6893, Fax: 82-62-232-6294, E-mail: shkwak@jnu.ac.kr This study was financially supported by Chonnam National University, 2008.

(c) This is an open-access article distributed under the terms of the Creative Commons Attribution Non-Commercial License (http:// creativecommons.org/licenses/by-nc/3.0/), which permits unrestricted non-commercial use, distribution, and reproduction in any medium, provided the original work is properly cited. 


\section{Introduction}

The stress response to surgery or inflammation is mediated by complex interactions. Cytokines, such as tumor necrosis factor alpha (TNF- $\alpha$ ), Interleukin (IL)-1, and IL-6, as well as other humoral mediators of inflammation, are potent activators of the central stress-responsive neurotransmitter systems.

The stress responses to surgery and inflammation activate a systemic inflammatory response characterized clinically by changes in a variety of functions, such as nervous, endocrine, immune, and hematopoietic systems [1]. The systemic inflammatory response is maintained by a range of factors, including cytokine production. The overproduction of pro-inflammatory cytokines and the inappropriate release of anti-inflammatory cytokines during surgery can harm the organs [2]. The proinflammatory cytokine, IL-6, is a sensitive marker of tissue injury, and has been associated with invasiveness and the duration of surgery [3].

Urinary trypsin inhibitor (UTI), a serine protease inhibitor, is used widely in patients with inflammatory disorders, including shock and pancreatitis. It suppresses the human leukocyte elasetase activity and inhibits the production of proinflammatory cytokines, such as TNF- $\alpha$ and IL-8 $[4,5]$.

The circulating concentration of pro-inflammatory cytokines, IL-6 and IL-8, were measured in patients undergoing a gastrectomy, with and without UTI infusion.

\section{Materials and Methods}

Thirty patients undergoing a gastrectomy under general anesthesia were studied. There were no differences in age, gender, weight, and height between the groups (Table 1). All patients provided written informed consent and the study was approved by the Institutional Review Board for Human Studies. Those scheduled to undergo a re-operation, as well as those with infectious diseases or a history of chronic obstructive

Table 1. Demographic Data, Time, Fluid and Blood Loss

\begin{tabular}{lcc}
\hline & $\begin{array}{c}\text { Control } \\
(\mathrm{n}=15)\end{array}$ & $\begin{array}{c}\text { UTI } \\
(\mathrm{n}=15)\end{array}$ \\
\hline Age (yr) & $54 \pm 7$ & $58 \pm 9$ \\
Weight (kg) & $61 \pm 9$ & $60 \pm 14$ \\
Sex (Male/Female) & $22 / 8$ & $20 / 10$ \\
Duration of Anesthesia (min) & $171 \pm 76$ & $179 \pm 27$ \\
Duration of surgery (min) & $156 \pm 76$ & $164 \pm 27$ \\
Fluid input (ml) & $2,920 \pm 645$ & $2,655 \pm 664$ \\
Blood loss (ml) & $580 \pm 185$ & $478 \pm 148$ \\
\hline
\end{tabular}

Values are reported as the mean \pm SD or number of patients. UTI: urinary trypsin inhibitor. pulmonary disease (COPD) were excluded.

After at least 8 hours of fasting, the participants were given midazolam $0.1 \mathrm{mg} / \mathrm{kg}$ orally, 1 hour before the induction of anesthesia. When the patients arrived at the operating room, a noninvasive blood pressure, ECG, pulse oximetry, and bispectral index monitor (BIS XP monitor A 2000, Aspect Medical System Inc., Natick, MA, USA) was attached. The arterial pressure was measured continuously through a 20 gauge catheter that had been inserted into the radial artery before the induction of general anesthesia. Anesthesia was induced using IV injections of $1 \%$ propofol $(2 \mathrm{mg} / \mathrm{kg}$ ) and rocuronium ( $1-2$ $\mathrm{mg} / \mathrm{kg}$ ). Anesthesia was maintained with sevoflurane and $50 \%$ $\mathrm{N}_{2} \mathrm{O}$ in oxygen. The sevoflurane level was adjusted so that the bispectral index would maintain a level of $40-60$.

After inducing anesthesia, the patients were assigned randomly to 2 groups; the UTI treatment group $(\mathrm{n}=15)$ and control group $(n=15)$. During surgery, the treatment group received 100,000 units of UTI intravenously over the following 2 hours, while nothing was given to the control group. To observe the changes in the level of inflammatory cytokines (IL-6 and IL-8), blood samples were taken from the radial artery before surgery (T0), 10 minutes after its onset (T1), at its end (T2), and 1 hour postsurgery (T3).

The patient's blood $(3 \mathrm{ml})$ was centrifuged at $4^{\circ} \mathrm{C}$ and the serum was stored at $-70^{\circ} \mathrm{C}$. The arterial pressure and heart rate at the time of sampling were recorded, and the amount of blood loss, volume of fluids and transfusions administered, total time under anesthesia, and duration of surgery were also recorded. In addition, liver function tests (aspartate transaminase [AST], alanine transaminase [ALT]) and renal function tests (blood urea nitrogen $[\mathrm{BUN}]$, creatinine [Cr]) were performed before and after surgery.

The IL-6 and IL-8 levels were measured using an Enzymelinked immunosorbent assay (ELISA) kit (R\&D system, Minneapolis MN, USA), and the values are expressed as the mean \pm SD. SPSS (version 15.0, SPSS Inc, Chicago, USA) was used for the statistics. An independent sample t-test was used to compare the demographic data, time, fluid, blood loss and laboratory data. The time series changes in the IL-6 levels were analyzed by repeated measure analysis within the groups and a MannWhitney test between the groups. A P value $<0.05$ was considered significant.

Group sample sizes of 13 and 13 were considered necessary to achieve an $80 \%$ power to detect a difference of 25.0 between the null hypothesis that both group means are 45.7 and the alternative hypothesis that the mean of group 2 is 20.7 with estimated group standard deviations of 26.8 and 10.5 and a significance level (alpha) of 0.05 using a two-sided Mann-Whitney test assuming a normal distribution. 


\section{Results}

The intraoperative characteristics, such as the duration of surgery and anesthesia, blood loss, and total amount of the fluid given, were comparable (Table 1). The serum concentrations of IL-6 were higher in both groups at T2 and T3 than at T0, but the degree of these increases was significantly smaller in the UTI group than in the control group. The serum concentrations of IL-8 increased significantly in the control group, but not in the UTI group at T2 and T3 compared with T0 (Table 2).

There was an increase in the postoperative hepatic enzymes involving AST and ALT compared to the preoperative values in the control group, but not in the UTI group (Table 3).

\section{Discussion}

The main result of this study is that UTI attenuates the production of IL- 6 by surgical stress.

The serum level of IL-6 is a sensitive marker of tissue damage. The level increased in response to surgical stimuli and the maximal level was affected by the duration of surgery [3]. IL-6 is related directly to the appearance of endotoxins. Endotoxin is referred to as lipopolysaccharide (LPS), which activates the complements or cytokines, and is an important cause of the systemic inflammatory responses [6]. In addition, IL-6 causes acute reactions in the liver [7], acts as an endogenous pyrogen [8], and stimulates the secretion of polymorphonuclear leukocyte elastase (PMNE), which causes sepsis or acute

Table 2. Changes in IL-6 and IL-8 (pg/ml)

\begin{tabular}{cccccc}
\hline \multirow{2}{*}{ Time } & \multicolumn{2}{c}{ IL-6 } & \multicolumn{2}{c}{ IL-8 } \\
\cline { 2 - 3 } \cline { 5 - 6 } & Control & UTI & & Control & UTI \\
\hline T0 & 0 & 0 & & 0 & $0.1 \pm 0.1$ \\
T1 & 0 & 0 & 0 & $0.2 \pm 0.2$ \\
T2 & $20.0 \pm 9.4^{*}$ & $10.3 \pm 4.2^{*, \dagger}$ & & $0.6 \pm 0.5^{*}$ & $1.2 \pm 1.7$ \\
T3 & $45.7 \pm 26.8^{*}$ & $20.7 \pm 10.5^{*, \dagger}$ & & $0.8 \pm 0.6^{*}$ & $1.7 \pm 3.1$ \\
\hline
\end{tabular}

Values are reported as the mean \pm SD. UTI: urinary trypsin inhibitor.

${ }^{*} \mathrm{P}<0.05$ versus $\mathrm{T} 0,{ }^{\dagger} \mathrm{P}<0.05$ versus the control. respiratory distress syndrome [9-11]. The reactions mentioned above are inhibited by UTI [11].

In humans and animal models of systemic inflammation, TNF- $\alpha$, IL- 6 and IL- 8 are released sequentially, causing inflammation cascades. IL- 1 and TNF- $\alpha$ can induce the expression of IL-8 in the transcription phase, in cells, such as the endothelial cells and monocytes in the blood [12-14]. This IL-8 promotes tissue injury by neutrophil infiltration and the secretion of the lysosomal enzymes along with the peroxide anions [15].

In this study, the post-operative IL-6 and IL-8 levels were higher than the levels checked before surgery. The postoperative IL-6 level was increased significantly in both groups but the degree of the increase in the treatment group was attenuated. The post-operative IL-8 level of the control group increased significantly, but the treatment group did not show such marked changes. In addition, there were no significant differences between the IL-8 levels in the two groups. The IL-8 levels have relatively large standard deviations and the mean values are $<2 \mathrm{pg} / \mathrm{ml}$. The mean minimum detectable dose of the IL-8 ELISA kit was $3.5 \mathrm{pg} / \mathrm{ml}$, which means that these IL-8 values should be rejected. Previous studies reported that the IL-8 levels ranged from several tens to several hundred after surgery, such as cardiac surgery and aortic surgery $[16,17]$. These results indicate some differences in the intensity of stress between procedures. The IL-8 levels are expected to increase along with IL- 6 because it also is an inflammatory cytokine. However, this was not observed in this study.

UTI is a glycoprotein stable in both heat and acids, and is a serine protease inhibitor found in human urine or blood. UTI is secreted when inter- $\alpha$-trypsin inhibitors are degraded by neutrophilic elastases [18]. UTI has many physiological effects, including the inhibition of neutrophilic elastase, trypsin, $\alpha$-chymotrypsin, plasmin, and cathepsin G. Previous studies showed that UTI inhibits proinflammatory substances, such as IL-8, which is induced by neutrophilic elastase in human bronchoepithelial cells [4]. Studies have also found that UTI inhibits TNF- $\alpha$ from monocytes, intracellular adhesion molecule-1 (ICAM-1) induced by LPS, and the infiltration of neutrophils in ischemic

Table 3. Laboratory Data

\begin{tabular}{|c|c|c|c|c|}
\hline & \multicolumn{2}{|c|}{ Control } & \multicolumn{2}{|c|}{ UTI } \\
\hline & Preoperative & Postoperative & Preoperative & Postoperative \\
\hline $\operatorname{WBC}\left(10^{3} / \mathrm{mm}^{3}\right)$ & $7.2 \pm 1.7$ & $14.6 \pm 4.6$ & $6.7 \pm 1.4$ & $13.4 \pm 6.3$ \\
\hline Hemoglobin (g/dl) & $13.0 \pm 2.0$ & $11.5 \pm 1.7$ & $14.0 \pm 1.8$ & $11.9 \pm 1.9$ \\
\hline AST (IU/L) & $19.4 \pm 5.5$ & $67.9 \pm 57.6^{*}$ & $28.5 \pm 12.3$ & $42.4 \pm 17.6$ \\
\hline ALT (IU/L) & $20.4 \pm 11.4$ & $66.8 \pm 58.8^{*}$ & $31.2 \pm 15.7$ & $67.5 \pm 79.2$ \\
\hline BUN (mg/dl) & $14.0 \pm 3.4$ & $14.5 \pm 4.3$ & $13.5 \pm 4.0$ & $13.9 \pm 3.7$ \\
\hline Creatinine $(\mathrm{mg} / \mathrm{dl})$ & $0.9 \pm 0.12$ & $0.9 \pm 0.2$ & $0.9 \pm 0.2$ & $1.1 \pm 0.5$ \\
\hline
\end{tabular}

Values are reported as the mean \pm SD. UTI: urinary trypsin inhibitor. $* \mathrm{P}<0.05$ versus the preoperative group. 
brain tissues $[5,19,20]$. UTI 100,000 units were used in this study. The routine clinical dose of UTI is 300,000 units [21], but a previous study showed that UTI 100,000 units was sufficient to attenuate the inflammation [22].

All patients were injected with fentanyl 100-150 $\mu \mathrm{g}$ intravenously for pain control after surgery. It has been suggested that large doses of opioids may suppress the cytokine response to surgery, but conventional doses of opioids do not [23]. Furthermore, the consumption of fentanyl in both groups was similar. Therefore, the influence of fentanyl on the results at T3 was not considered.

Interestingly, the liver enzymes (AST, ALT) were elevated after surgery in the control group, but not in the UTI treatment group. The rise in liver enzymes indicates an intra-hepatic cause of liver dysfunction. The common etiologies of hepatic dysfunction in the perioperative period include sustained hypoxia or hypotension resulting in decreased hepatic blood flow, direct toxicity from drugs, hemolysis, and a worsening of preexisting liver disease [24]. These results showed that UTI must have had an effect on the post-operative liver enzyme levels.

In conclusion, UTI reduces the inflammatory responses to surgical stress by inhibiting IL-6 activation. However, further studies will be needed to examine its effect on the liver functions.

\section{References}

1. Angele MK, Faist E. Clinical review: immunodepression in the surgical patient and increased susceptibility to infection. Crit Care 2002; 6: 298-305.

2. Partrick DA, Moore EE, Moore FA, Biffl WL, Barnett CC Jr. Release of anti-inflammatory mediators after major torso trauma correlates with the development of postinjury multiple organ failure. Am J Surg 1999; 178: 564-9.

3. Biffl WL, Moore EE, Moore FA, Peterson VM. Interleukin-6 in the injured patient. Marker of injury or mediator of inflammation? Ann Surg 1996; 224: 647-64.

4. Nakamura H, Abe S, Shibata Y, Sata M, Kato S, Saito H, et al. Inhibition of neutrophil elastase-induced interleukin-8 gene expression by urinary trypsin inhibitor in human bronchial epithelial cells. Int Arch Allergy Immunol 1997; 112: 157-62.

5. Aosasa S, Ono S, Mochizuki H, Tsujimoto H, Ueno C, Matsumoto A. Mechanism of the inhibitory effect of protease inhibitor on tumor necrosis factor alpha production of monocytes. Shock 2001; 15: 101-5.

6. Pallua N, Low JF, von Heimburg D. Pathogenic role of interleukin-6 in the development of sepsis. Part II: significance of anti-interleukin-6 and anti-soluble interleukin-6 receptor-alpha antibodies in a standardized murine contact burn model. Crit Care Med 2003; 31: 1495-501.

7. Geiger T, Andus T, Klapproth J, Hirano T, Kishimoto T, Heinrich PC. Induction of rat acute-phase proteins by interleukin 6 in vivo. Eur J Immunol 1988; 18: 717-21.
8. Cartmell T, Poole S, Turnbull AV, Rothwell NJ, Luheshi GN. Circulating interleukin- 6 mediates the febrile response to localised inflammation in rats. J Physiol 2000; 526: 653-61.

9. Kodama T, Yukioka H, Kato T, Kato N, Hato F, Kitagawa S. Neutrophil elastase as a predicting factor for development of acute lung injury. Intern Med 2007; 46: 699-704.

10. Johnson JL, Moore EE, Tamura DY, Zallen G, Biffl WL, Silliman CC. Interleukin-6 augments neutrophil cytotoxic potential via selective enhancement of elastase release. J Surg Res 1998; 76: 91-4.

11. Inoue K, Takano H, Sato H, Yanagisawa R, Yoshikawa T. Protective role of urinary trypsin inhibitor in lung expression of proinflammatory cytokines accompanied by lethal liver injury in mice. Immunopharmacol Immunotoxicol 2009; 31: 446-50.

12. Cohen J. The immunopathogenesis of sepsis. Nature 2002; 420: 885 91.

13. Matsushima K, Morishita K, Yoshimura T, Lavu S, Kobayashi Y, Lew $\mathrm{W}$, et al. Molecular cloning of a human monocytederived neutrophil chemotactic factor (MDNCF) and the induction of MDNCF mRNA by interleukin 1 and tumor necrosis factor. J Exp Med 1988; 167: 1883-93.

14. Mukaida N, Mahe Y, Matsushima K. Cooperative interaction of nuclear factor-kappa B- and cis-regulatory enhancer binding protein-like factor binding elements in activating the interleukin-8 gene by pro-inflammatory cytokines. J Biol Chem 1990; 265: 2112833.

15. Sekido N, Mukaida N, Harada A, Nakanish I, Watanabe Y, Matsushima K. Prevention of lung reperfusion injury in rabbits by a monoclonal antibody against interleukin-8. Nature 1993; 365: 654-7.

16. Kawamura T, Inada K, Okada H, Okada K, Wakusawa R. Methylprednisolone inhibits increase of interleukin 8 and 6 during open heart surgery. Can J Anaesth 1995; 42: 399-403.

17. Raijmakers PG, Groeneveld AB, Rauwerda JA, Schneider AJ, Teule GJ, Hack CE, et al. Transient increase in interleukin-8 and pulmonary microvascular permeability following aortic surgery. Am J Respir Crit Care Med 1995; 151: 698-705.

18. Hirose J, Ozawa T, Miura T, Isaji M, Nagao Y, Yamashiro K, et al. Human neutrophil elastase degrades inter-alpha-trypsin inhibitor to liberate urinary trypsin inhibitor related proteins. Biol Pharm Bull 1998; 21: 651-6.

19. Inoue K, Takano H, Yanagisawa R, Sakurai M, Shimada A, Yoshino S, et al. Protective role of urinary trypsin inhibitor in acute lung injury induced by lipopolysaccharide. Exp Biol Med 2005; 230: 281-7.

20. Yano T, Anraku S, Nakayama R, Ushijima K. Neuroprotective effect of urinary trypsin inhibitor against focal cerebral ischemiareperfusion injury in rats. Anesthesiology 2003; 98: 465-73.

21. Aibiki M, Cook JA. Ulinastatin, a human trypsin inhibitor, inhibits endotoxin-induced thromboxane B2 production in human monocytes. Crit Care Med 1997; 25: 430-4.

22. Yoo JW, Ryu JK, Lee SH, Woo SM, Park JK, Yoon WJ, et al. Preventive effects of ulinastatin on post-endoscopic retrograde cholangiopancreatography pancreatitis in high-risk patients: a prospective, randomized, placebo-controlled trial. Pancreas 2008; 37: 366-70.

23. Taylor NM, Lacoumenta S, Hall GM. Fentanyl and the interleukin-6 response to surgery. Anaesthesia 1997; 52: 112-5.

24. Hines RL, Marschall KE. Anesthesia and co-existing disease. 5th ed. Philadelphia, Churchill Livingstone. 1993, pp 276-8. 